\section{Germination of Encapsulated Somatic Embryos of Cyclamen persicum}

\author{
Traud Winkelmann, ${ }^{1}$ Lara Meyer, and Margrethe Serek \\ Institute of Floriculture, Tree Nursery Science and Plant Breeding, Section \\ Floriculture, University of Hannover, Herrenhaeuser Str. 2, D-30419 Hanover, \\ Germany
}

Additional index words. alginate, artificial seeds, hollow beads, synthetic seeds, storage type of capsules. Although the term "hollow" is not precisely chosen, since it is defined as being empty, in the present study it will be used in accordance to Patel et al. (2000).

The objectives of this study were 1 ) to compare two encapsulation methods, conventional alginate beads versus hollow beads (Patel et al., 2000), for cyclamen somatic embryos, 2) to analyse their effects on germination before and after storage at $4{ }^{\circ} \mathrm{C}$, and 3 ) to test the influence of adding nutrients to the capsules on somatic embryo development on medium or mediumfree supports.

\section{Materials and methods}

Somatic embryo production for encapsulation. Somatic embryos were produced in liquid cultures. Embryogenic callus was induced following the protocol of Schwenkel and Winkelmann (1998) from ovules of the genotype 3738-14 (a seedling of 'Purple Flamed'). Suspension cultures were established and maintained according to Winkelmann et al. (1998). Fourteen days after subculture the size fraction 500 to $1000 \mu \mathrm{m}$ was transferred to liquid hormone-free medium(Hohe et al., 2001) at a density of $1 \%$ PCV (packed cell volume). Somatic embryos differentiated within 2 to 3 weeks cultured at $120 \mathrm{rpm}$ and 23 to $25^{\circ} \mathrm{C}$ in darkness and were collected on a sieve of $500 \mu \mathrm{m}$. These embryos mainly at globular stage were used for the encapsulation experiments.

Encapsulation in conventional alginate beads. Somatic embryos were singly sucked up within $50 \mu \mathrm{L}$ of a sodium alginate solution using a micropipette with a cut tip to provide a wider opening. Initial tests using sodium alginate concentrations of $1.0 \%, 1.5 \%, 2.0 \%$, and $2.5 \%$ showed that $1.5 \%$ sodium alginate solution gave the best result. The $50-\mu \mathrm{L}$ droplets each containing one selected somatic embryo were dropped into a calcium chloride solution (68 $\mathrm{mm}$ ), which was stirred for $20 \mathrm{~min}$ to induce polymerisation and bead formation. After two washes in deionized water of $10 \mathrm{~min}$ each alginate beads were collected on a sieve (1000 $\mu \mathrm{m})$ and used for storage or germination tests (see below). All solutions used for encapsulation were autoclaved (Patel et al., 2000)

Encapsulation in hollow beads. Somatic embryos were suspended in $1.5 \%$ carboxymethylcellulose solution (containing $1 \mathrm{~g} \mathrm{CaCl}_{2} \times 2$ $\mathrm{H}_{2} \mathrm{O}$ in $100 \mathrm{~mL}$ ) in a petri dish. Single embryos were taken up within $35 \mu \mathrm{L}$ of this solution using a micropipette (as described for alginate beads above) and dropped into $0.8 \%$ sodium alginate solution under slow stirring. After 20 min the alginate solution was diluted by adding the double amount of water. After another 10 min the alginate solution was discarded and the beads were washed for two times in water ( 5 min each). Finally the surface of the beads was hardened by replacing the water with $\mathrm{CaCl}_{2}(68$ mM) (Patel et al. 2000). The further steps were same as for alginate beads.

Experimental setup. To test the two different encapsulation methods regarding development and germination of somatic embryos the first experiment was performed. Somatic embryos encapsulated into either conventional alginate 
beads or hollow beads were compared to control (nonencapsulated) embryos.

To test the storability of control and encapsulated somatic embryos they were kept for four weeks at $4{ }^{\circ} \mathrm{C}$ in sealed petri dishes on moist filter paper (under sterile conditions). The somatic embryos for these capsules as well as for controls were derived from the same liquid cultures that had been used for the first experiment.

Aim of the third experiment was to investigate the effect of incorporating nutrients into the capsules. For this purpose the solutions in which the embryos were sucked up for encapsulation were prepared with Ca-free hormonefree $1 / 2$ MS medium instead of water. Alginate beads and hollow beads containing nutrients or not were then cultured on the normal cyclamen differentiation medium $\left(1 / 2 \mathrm{MS}\right.$ with $30 \mathrm{~g} \cdot \mathrm{L}^{-1}$
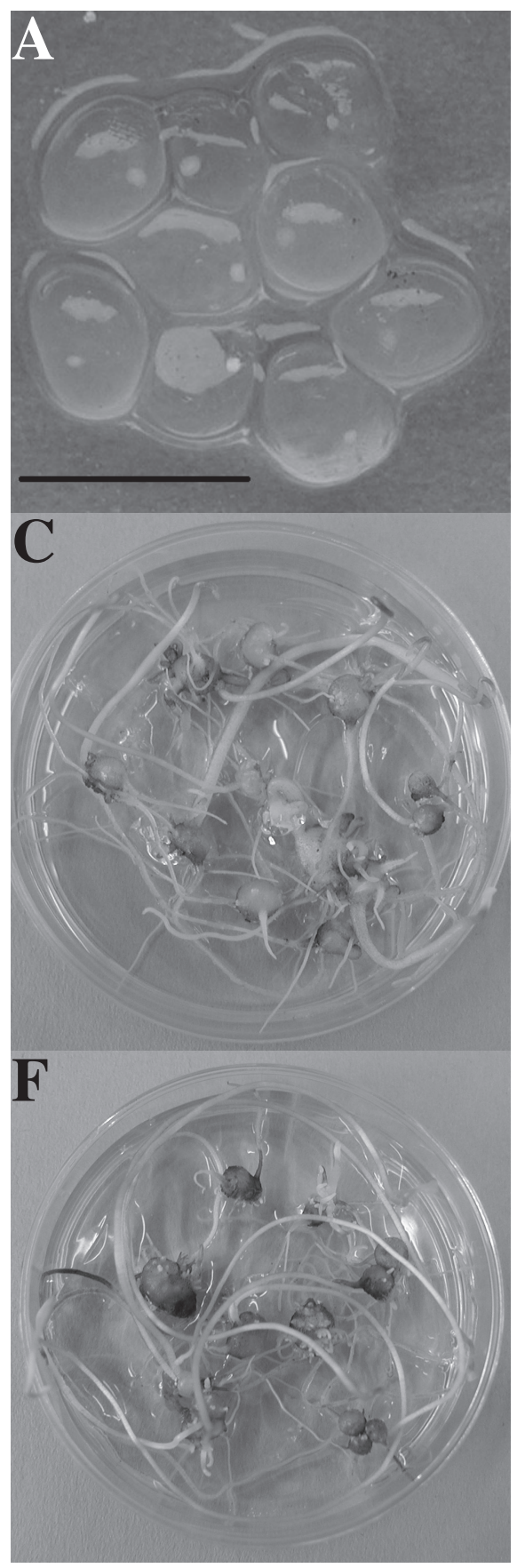

sucrose and $2 \mathrm{~g} \cdot \mathrm{L}^{-1}$ glucose, Schwenkel and Winkelmann, 1998) or on water solidified with $8.0 \mathrm{~g} \cdot \mathrm{L}^{-1}$ agar (SERVA 11396).

Ten embryos each either control or encapsulated were placed in 6-cm plastic petri dishes containing hormone-free differentiation medium (modified $1 / 2 \mathrm{MS}$ medium as described by Schwenkel and Winkelmann, 1998) and cultivated at $20{ }^{\circ} \mathrm{C}$ in complete darkness. Development of somatic embryos and germination were evaluated in 4-week intervals. Somatic embryos were scored as germinated, when the cotyledon came out of the capsule, since in cyclamen incomplete germination resulting in formation of only roots and sometimes small tubers was observed. Each experiment was
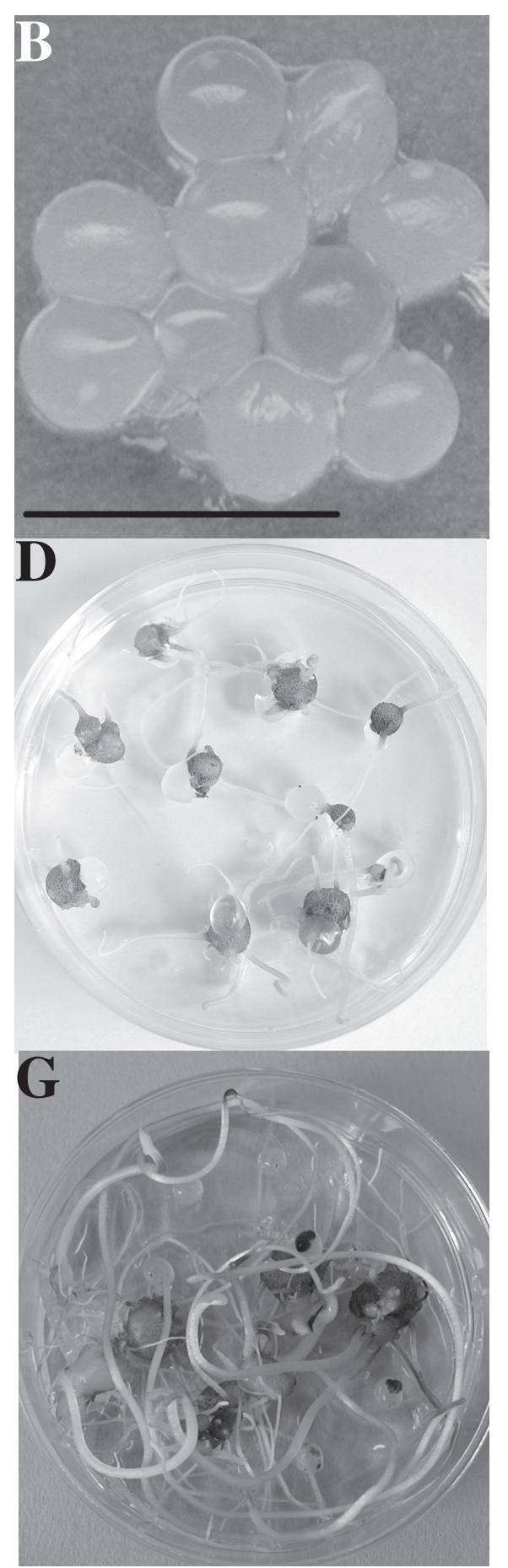

repeated twice with three to six petri dishes per treatment.

Germination is presented in the figures in percentages and the means and standard deviations between the treatments were compared.

\section{Results}

Initial experiments revealed that alginate at a concentration of $1.5 \%$ and higher resulted in spherical and solid beads (Fig. 1B), while at $1.0 \%$ the shape of the capsules was irregular and handling with forceps not easy. Therefore, for all subsequent experiments alginate beads were prepared using $1.5 \%$ sodium alginate solution. During the preparation of hollow beads it was observed that in some experiments the beads were destroyed during the last steps of production making this method difficult for practical application. In these cases only half of the capsules could be included in the

Fig. 1. Encapsulated somatic embryos of cyclamen ( $\mathbf{C}$ to $\mathbf{H}$ in 6-cm petri dishes). (A) Hollow beads directly after preparation (the bar $=1 \mathrm{~cm})$. $(\mathbf{B})$ Alginate beads directly after preparation bar = $1 \mathrm{~cm}$ ). (C) Germinating control embryos (not encapsulated) after 2 months of culture. (D) Germination from in alginate beads after 2 months of culture. (E) Germination from hollow beads after 2 months of culture.(F) Germinating control embryos (not encapsulated) after 4 months of culture. (G) Germination from alginate beads after 4 months of culture. (H) Germination from hollow beads after 4 months of culture.
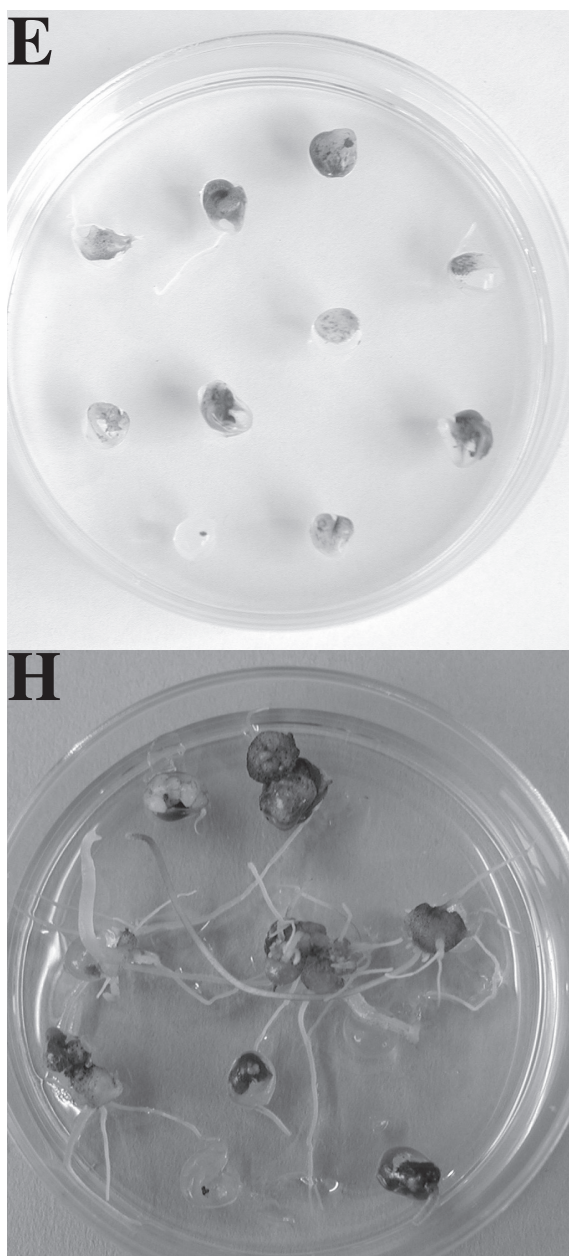
experiments. Since this problem could not be overcome, research in standardizing the structure and consistency of the beads seems to be necessary.

When the two types of capsules were compared, it was observed that somatic embryos were often not centrally placed in alginate beads (Fig. 1B), while in hollow beads they were totally covered (Fig. 1A). In hollow beads the formation of tubers, roots and cotyledons took place within the capsule in contrast to conventional alginate beads where somatic embryos expanded very early and soon grew out of the capsules (Fig. $1 \mathrm{D}$ and $\mathrm{E})$. Germination was asynchronous in all variants. For control embryos the final germination of about $97 \%$ was reached late, after 24 weeks (Fig. 2). However, this high percentage points to the fact that somatic embryos of good quality have been produced in the liquid culture system(Fig. 1CandF). Somatic embryos encapsulated in conventional alginate beads were not negatively affected by the encapsulation, since they also reached final germination percentages of $97 \%$, but their development was slower (Fig. 1D and G and Fig. 2). Often they formed bigger tubers with many shoot buds. In contrast, embryos in hollow beads did not reach final germination percentages as high as control or alginate encapsulated ones (Fig. 2). Their development was much slower and after the first increase in size many embryos turned brown and did not break through the capsules (Fig. 1Eand H). In addition the variation between the two replications was pronounced in case of hollow beads.

To test the ability of somatic embryos to germinate after four weeks of storage at $4{ }^{\circ} \mathrm{C}$ the second experiment was performed. Four weeks after the embryos had been placed at $20{ }^{\circ} \mathrm{C}$ for germination first control embryos showed formation of cotyledons (Fig. 3). However, their development was slower than that of unstored embryos and only $68 \%$ were able to germinate. Again alginate beads lead to similar final germination percentages as compared to controls, but these were reached with a delay (Fig. 3). In contrast, somatic embryos in hollow beads only expressed very poor germination after cool storage.

To test the effect of additional nutrients and carbohydrates within the capsules liquid hormone-free medium has been integrated into the capsules. For both, alginate beads as well as hollow beads, the medium capsules resulted in better and earlier germination when compared to capsules without medium. This becomes obvious from the steeper rise of the curves and the higher final germination percentages observed for the medium-containing beads in Fig. 4.

The final aim for application of synthetic seeds is the direct sowing into soil. Therefore we tried to germinate encapsulated embryos on water agar ( $0.8 \%$ agar dissolved in water) containing neither nutrients nor carbohydrates. The control embryos did not germinate without medium neither did somatic embryos encapsulated without medium. Also embryos in capsules prepared with medium did not show any development, but turned brown and died indicating that the small amount of nutrients within 50 or $35 \mu \mathrm{L}$ of medium (see materials and methods) did not support growth.

\section{Discussion}

In this study it was shown, that somatic embryos of cyclamen can be encapsulated in alginate beads without losing germination ability. Only a minor delay in germination was observed probably due to the mechanical barriers. Likewise in some other plant species alginate has proven to be suitable for encapsulating somatic embryos leading to germination similar to that of control embryos, e.g., in pelargonium (Gill et al., 1994) or Camellia japonica (Janeiro et al., 1997). Castillo et al. (1997) reported on pronounced differences between two types of sodium alginate derived from different manufacturers and demonstrated that for Carica papaya alginate beads the time of hardening in $\mathrm{CaCl}_{2}$ was important as well. The composition of alginate can vary and especially the ratio of guluronic and mannuronic acids (Redenbaugh et al., 1993) is important for the applicability in plant cell culture. Since alginate is a product of natural origin, impurities having phytotoxic effects can not be ruled out.

There are only few reports available dealing with alternative coating materials. One recent comprised hollow beads for encapsulation of plant cell material and embryos (Patel et al., 2000). In our studies one advantage of hollow beads was the complete protection of the embryos that were more centrally placed in the capsules. The first steps of development took place within the capsules, which, with regard to the final aim of synthetic seeds, is positive as well. But since some embryos turned brown and were not able to break through the outer capsule wall, this type of encapsulation has to be improved for cyclamen. In the study of Patel et al. (2000) carrot seeds germinated at $100 \%$ within the hollow beads, which was better than control seeds, but only $13 \%$ were able to grow out of the capsules.

Storage of cyclamen somatic embryos at $4{ }^{\circ} \mathrm{C}$ resulted in a decrease in the germination rates for both control and encapsulated ones (Fig. 3). The cool temperatures may be one reason for this, the lack of nutrients during storage could be another. However, in other species somatic embryos encapsulated in alginate beads were able to tolerate storage at low temperatures better than controls. In Carica papaya capsules were stored at $10{ }^{\circ} \mathrm{C}$ for 85 days and some embryos survived, while naked somatic embryos did not (Castillo et al., 1997). Similar observations were made for Camellia japonica where storage at $4{ }^{\circ} \mathrm{C}$ reduced the germination frequency stronger in nonencapsulated somatic embryos (Janeiro et al., 1997). Synthetic seeds of Clitoria ternatea could be stored at $2{ }^{\circ} \mathrm{C}$ for $30 \mathrm{~d}$ without reduction in viability, whereas control embryos only showed $4 \%$ viability after cool storage (Malabadi and Nataraja, 2002). One of the main problems in storing alginate capsules in the hydrated form is the poor respiration of encapsulated tissues (Redenbaugh et al., 1993). Therefore storage of alginate encapsulated somatic embryos in a dried form has been suggested and was carried out successfully for carrot (Liu et al., 1992). Timbert et al. (1996) tested different additives to alginate, which resulted in higher water activities in the synthetic seeds during dehydration. Addition of kaolin or gellan gum lead to slower dehydration being beneficial for the somatic embryos. With regard to the rehydration process the authors stress that rehydration has to be slow, but sufficient to promote regrowth. In case of cyclamen first results on desiccation of naked somatic embryos are promising (Winkelmann et al., 2003), so that a combination of encapsulation and dehydration should be tested in this species as well.

The use of alginate and carboxymethylcellulose dissolved in nutrient medium resulted

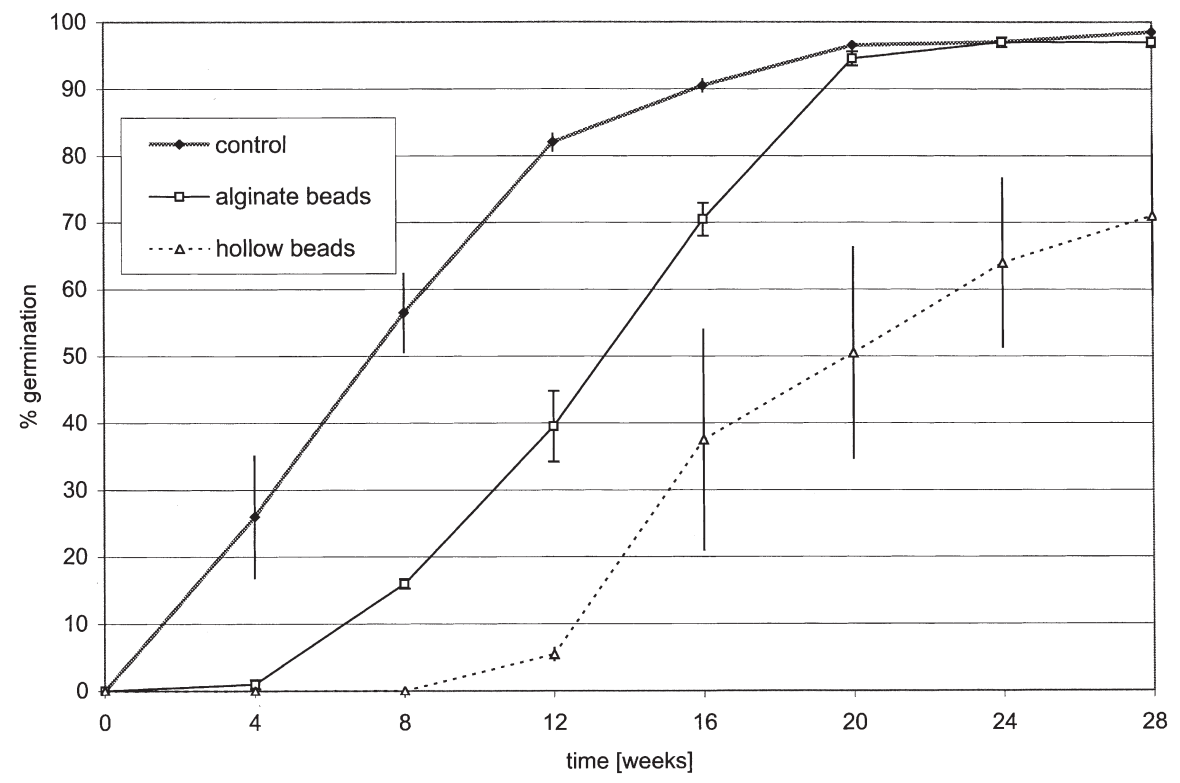

Fig. 2. Germination of control (not encapsulated) somatic embryos and embryos in conventional alginate beads and in hollow beads (average and standard deviation of two experiments). 


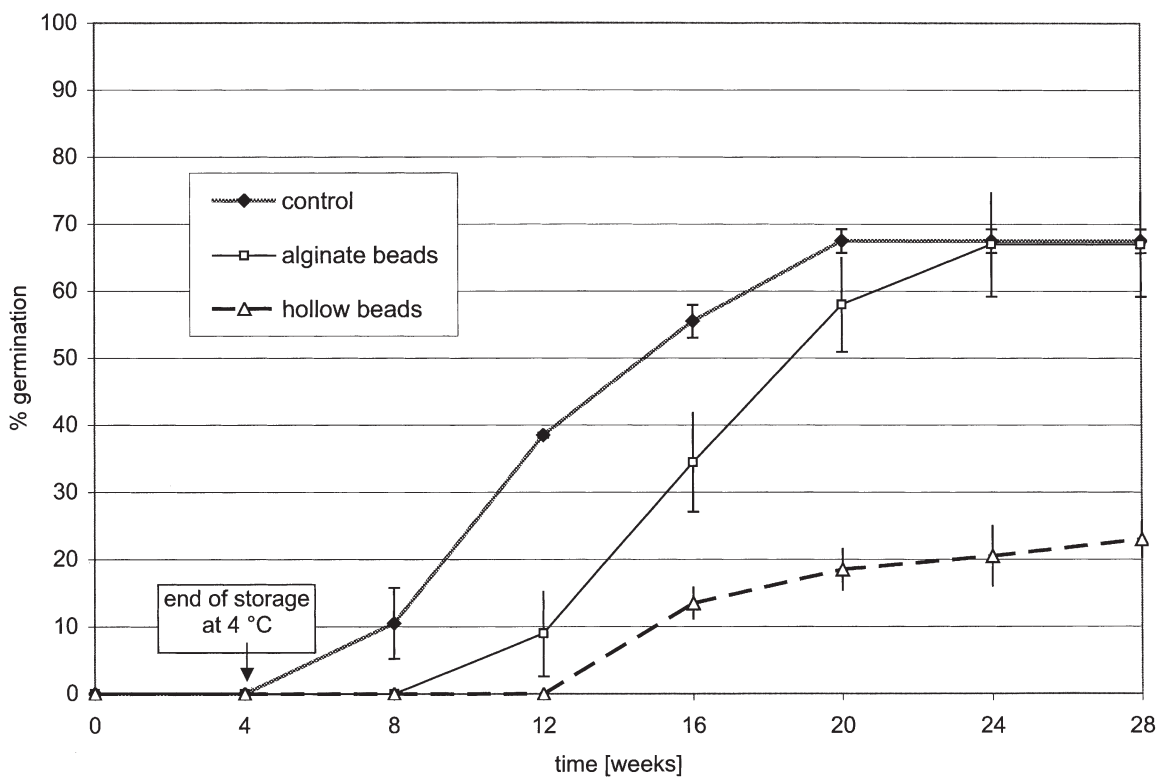

Fig. 3. Germination of control (not encapsulated) somatic embryos and embryos in conventional alginate beads and in hollow beads after 4 weeks storage at $4{ }^{\circ} \mathrm{C}$ (average and standard deviation of two experiments).

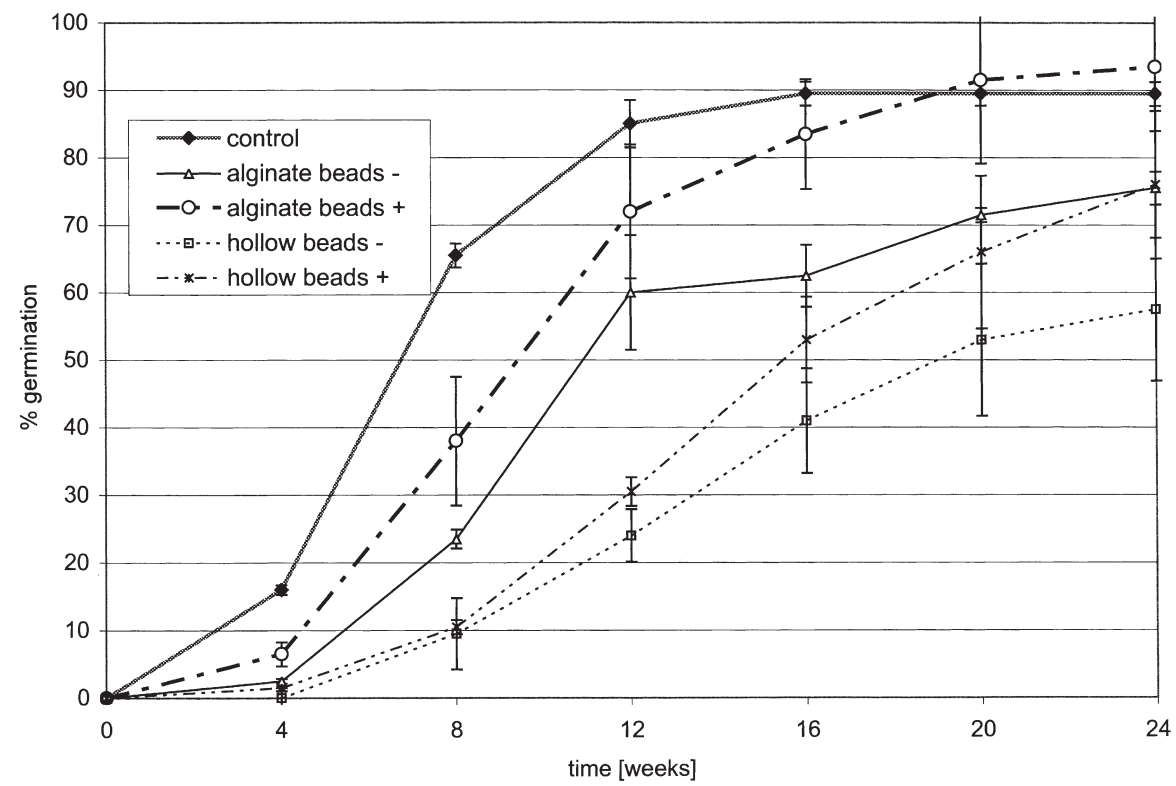

Fig. 4. Germination of somatic embryos in different capsule types prepared with (+) or without (-) medium in comparison to controls (all variants were cultured on hormone-free medium for germination).

in better germination (Fig. 4). On the other hand this integration of medium into the capsules was not sufficient to promote growth on water agar. Somatic embryos of tangerine placed on medium free supports also did not germinate, even if they had been encapsulated with medium (Nieves et al., 1998). The fact that embryos encapsulated without medium but cultured on medium did germinate shows that diffusion of nutrients through the capsules takes place. Therefore it could also be possible that nutrients are lost from the capsules with medium by diffusion into the water agar.

If the technique of synthetic seeds is going to be integrated in horticultural practice the encapsulated embryos should be capable to germinate in the soil. Two strategies can be applied to reach this aim. On the one hand a kind of artificial endosperm can be developed which supports germination and is protected against leaching. Possible supplements for an artificial endosperm could be sucrose in form of microcapsules (Mamiya and Sakamoto 2001; Onishi et al., 1994) or amino acids (Nieves et al., 1998). On the other hand culture conditions can be modified so that encapsulatable units are produced, which are able to germinate without external supply of carbohydrates and nutrients. The concept of developing encapsulatable units has been applied for celery and carrot (Onishi et al., 1994) and for asparagus (Mamiya and Sakamoto, 2001). 


\section{Literature Cited}

Bongartz, W. 1999. Cyclamen. Thalacker-Verlag Braunschweig.

Castillo, B., M.A.L. Smith, and U.L. Yadava. 1997. Plant regeneration from encapsulated somatic embryos of Carica papaya L. Plant Cell Rpt. 17:172-176.

Dupuis J.M., C. Roffat, R.T. DeRose, and F. Molle. 1994. Pharmaceutical capsules as a coating system for artificial seeds. BioTechnology 12:385-389.

Fujii, J.A.A., D. Slade, and K. Redenbaugh. 1989. Maturation and greenhouse planting of alfalfa artificial seeds. In Vitro Cell. Dev. Biol. 25:1179-1182.

Gill, R., T. Senaratna, and P.K. Saxena. 1994. Thidiazuron-induced somatic embryogenesis enhances viability of hydrogel-encapsulated somatic embryos of geranium. J. Plant Physiol. 143:726-729.

Gray, D.J. 1989. Synthetic seed for clonal production of crop plants, p. 29-45. In: R.B. Taylorson (ed.). Recent advances in the development and germination of seeds. Plenum Press New York.

Hohe, A., T. Winkelmann, and H.G. Schwenkel. 1999a. $\mathrm{CO}_{2}$ accumulation in bioreactor suspension cultures of Cyclamen persicum Mill. and its effect on cell growth and regeneration of somatic embryos. Plant Cell Rpt. 18:863-867.

Hohe, A., T. Winkelmann, and H.G. Schwenkel. 1999b. The effect of oxygen partial pressure in bioreactors on cell proliferation and subsequent differentiation of somatic embryos of Cyclamen persicum. Plant Cell Tiss. Org. Cult. 59:39-45.

Hohe,A., T.Winkelmann, and H.G. Schwenkel. 2001. Development of somatic embryos of Cyclamen persicum Mill. in liquid culture. Gartenbauwissenschaft 66:219-224.

Janeiro, L.V., A. Ballester, and A.M. Vieitez. 1997. In vitro response of encapsulated somatic embryos of camellia. Plant Cell Tiss. Org. Cult. 51:119-125

Kitto, S. and J. Janick. 1985. Production of synthetic seeds by encapsulating asexual embryos of carrot. J. Amer. Hort. Soc. 119:277-282.

Liu, J.R., J.H. Jeon, S.G. Yang, H.S. Lee, N.H. Song, and W.J. Jeong. 1992. Dry type of carrot (Daucus carota L.) artificial seeds. Scientia Hort. 51:1-11.

Malabadi, R.B. and K. Nataraja. 2002. In vitro storage of synthetic seeds in Clitoria ternatea Linn. Phytomorphology 52:231-237.

Mamiya, K. and Y. Sakamoto. 2001. A method to produce encapsulatable units for synthetic seeds in Asparagus officinalis. Plant Cell Tiss. Org. Cult. 64:27-32.

Murashige, T. 1977. Plant cell and organ cultures as horticultural practices. Acta Hort. 78:17

Nieves, N.,J.C.Lorenzo, M.M. Blanco, J. Gonzalez, H. Peralta, M.Hernandez, R. Santos, O. Concepcion, C.G. Borroto,E. Borroto, R. Tapia, M.E. Martinez, Z. Fundora, and A. de los Gonzalez. 1998. Artificial endosperm of Cleopatra tangerine zygotic embryos: A model for somatic embryo encapsulation. Plant Cell Tiss. Org. Cult. 54:77-83.

Onishi, N., Y. Sakamoto, and T. Hirosawa. 1994 Synthetic seeds as an application of mass production of somatic embryos. Plant Cell Tiss. Org. Cult. 39:137-145.

Patel, A.V., I. Pusch, G. Mix-W., and K.D. Vorlop. 2000. A novel encapsulation technique for the production of artificial seeds. Plant Cell Rpt. 19:868-874
Pueschel,A.K.,H.G. Schwenkel, and T. Winkelmann. 2003. Inheritance of the ability for regeneration via somatic embryogenesis in Cyclamen persicum Plant Cell Tiss. Org. Cult. 72:43-51.

Redenbaugh, K., B. Paasch, J. Nichol, M. Kossler, P. Viss, and K. Walker. 1986. Somatic seeds: encapsulation of asexual plant embryos. Bio/ Technology 4:797-801.

Redenbaugh, K., J.A.A. Fujii, and D. Slade. 1993. Hydrated coatings for synthetic seeds, . p. 35-46. In: K. Redenbaugh (ed.). Synseeds. CRC Press, Boca Raton, Fla.

Schwenkel, H.G. 2001. Reproduction of Cyclamen persicum Mill. through somatic embryogenesis using suspension culture systems. Euro. Communities 2001 Rpt. COST Action 822, WG 2, p. 3-7.

Schwenkel, H.G. and T. Winkelmann. 1998. Plant regeneration via somatic embryogenesis from ovules of Cyclamen persicum Mill. Plant Tiss. Cult. Biotechnol. 4:28-34.

Timbert, R., J.N. Barbotin, and D. Thomas. 1996 Enhancing carrot somatic embryos survival during slow dehydration, by encapsulation and control of dehydration. Plant Sci. Limerick 120:215-222.

Winkelmann, T.,A. Hohe, and H.G. Schwenkel. 1998. Establishing embryogenic suspension cultures in Cyclamen persicum 'Purple Flamed'. Adv. Hort. Sci. 12:25-30.

Winkelmann, T., A. Hohe, A.K. Pueschel, and H.G. Schwenkel. 2000. Somatic embryogenesis in Cyclamen persicum MILL. Current Topics Plant Biol. 2:52-62.

Winkelmann, T., L. Meyer, and M. Serek. 2003. Maturation and desiccation of somatic embryos of Cyclamen persicum Mill. Acta Hort. 612:27-34 